\title{
Seeking the ultimate bowel preparation for colonoscopy: Is the end in sight?
}

\author{
Robert J Hilsden MD PhD FRCPC
}

\author{
Doc, I had to quit drinking that stuff, \\ it was giving me diarrhea.
}

$P_{\mathrm{in}}^{\mathrm{in}}$ ity the poor bowel prep researchers. Since colonoscopy was first introduced, they have undertaken the Herculean task of trying to design the means to vigorously and completely cleanse the colon in a way that is pleasant and tolerable for the patient. Over the same time, the expectations of endoscopists have increased: it is no longer adequate for the colon to merely be 'clean', it must be pristine. Any residual stool could hide a flat adenoma. The patients undergoing colonoscopy have also changed. Many are not patients at all, but instead, are healthy, asymptomatic individuals undergoing colonoscopy as a screening test for colorectal cancer. The bowel preparation is not only unpleasant, but it is frequently inconvenient, adding to the time lost from work or normal activities that results from undergoing a colonoscopy. Finally, a truly huge number of colonoscopies are performed each year (1). Even rare complications are of concern. Therefore, the bowel prep researcher seeks the perfect prep: one that immaculately cleanses the colon while being pleasant and convenient for the patient, and having an infinitesimally small risk of complications.

In the current issue of The Canadian Journal of Gastroenterology, two articles add to our knowledge in this area. Given the growing concern about missed neoplastic lesions in the right colon, the article by Kao et al (2) (pages 657-662) is timely. The authors report a randomized controlled trial of four bowel cleansing regimens: $4 \mathrm{~L}$ of polyethylene glycol (PEG) over a $4 \mathrm{~h}$ period; $2 \mathrm{~L}$ of PEG + bisacodyl; two $45 \mathrm{~mL}$ doses of sodium phosphate; and Pico-Salax (Ferring Pharmaceuticals Inc, Canada) plus $300 \mathrm{~mL}$ of magnesium citrate. A split-dose protocol, with some of the prep taken on the day of the colonoscopy, was used for those undergoing an afternoon colonoscopy. Although the authors do report a statistically significant difference between the bowel preps in the total score and the score for the right colon, it appears to be largely driven by the oral sodium phosphate preparation, which had the worst scores and is no longer on the market. The scores for the other three preps were quite similar, with a mean Ottawa Bowel Preparation Scale score for the right colon of 1.35, 1.10 and 1.37 for 4 L PEG, bisacodyl + 2 L PEG and Pico-Salax plus magnesium citrate, respectively (a score of 0 is excellent). The differences between the preparations only held true for colonoscopies performed in the morning, although, here again, the differences between the three commercially available preparations were small. However, the quality of the bowel preparations were generally better in the afternoon procedures compared with the morning procedures, and the scores for the right colon were consistently worse than those for the distal colon. Symptoms, including nausea, vomiting, abdominal cramps and bloating, were commonly reported for all bowel preparations.

The article by Vanner and Hookey (3) (pages 663-666) describes what the patient experiences after undergoing a sodium picosulphate/ magnesium citrate and bisacodyl regimen. Patients frequently ask what to expect when they are taking the bowel prep. Can they do this? Can they go there? Beyond telling them that they are going to experience significant diarrhea that will last several hours, I am usually at a loss to give them more specifics (fortunately having no personal experience to draw on). The authors had 100 patients keep a diary of their bowel preparation experiences. Bisacodyl $10 \mathrm{mg}$ was taken in the evening on the second and third days before the colonoscopy. Patients whose colonoscopy was scheduled for after 11:00 took one dose of Pico-Salax at 19:00 the evening before the colonoscopy and the second dose at 06:00 on the day of the colonoscopy. Patients who were scheduled before 11:00, took both doses the evening before at 17:00 and 22:00, respectively. The authors report that the first bowel movement occurred approximately $8 \mathrm{~h}$ to $9 \mathrm{~h}$ after each dose of bisacodyl, and the total number of bowel movements averaged approximately 3.5 per day. After the first dose of Pico-Salax, the average time to first bowel movement was $1.5 \mathrm{~h}$, and patients experienced a mean of 4.4 bowel movements over the next $4 \mathrm{~h}$. Patients taking the second dose of Pico-Salax at 22:00 had, on average, 6.5 bowel movements over the next $10 \mathrm{~h}$. Those taking the second dose at 06:00 had, on average, 4.5 bowel movements over the next $4 \mathrm{~h}$. Vanner and Hookey also found that the total number of bowel movements was positively associated with the quality of the bowel cleansing.

So, what lessons from these studies can I apply to my practice setting? The bulk of my time is spent in a community endoscopy centre that performs approximately 1000 colorectal cancer screening-related colonoscopies each month. As with any busy endoscopy unit, the quality of the bowel preparation is critically important. Preparations that are poorly tolerated or result in inadequate cleansing waste resources because they result in no-shows and cancelled appointments, procedures that need to be rescheduled and surveillance colonoscopies performed at shorter intervals than recommended by clinical practice guidelines. The primary message I take from the study by Kao et al (2) is that commercially available bowel preparations perform generally well, without marked differences with respect to the quality of colon cleansing. Even in terms of tolerability, they were quite similar, with only a 0.56 unit difference between the best and worst tolerated preps measured using a 7-point scale. It appears that all of the randomized patients were able to complete the bowel preparation and undergo their colonoscopy, with only four procedures being incomplete due to poor prep. Therefore, I do not see a clear winner in these results that would prompt me to change the current bowel preparation regimen (ie, split-dose PEG) at the Forzani \& McPhail Colon Cancer Screening Centre (Calgary, Alberta).

For patients undergoing a Pico-Salax/bisacodyl regimen, I can now provide better information about what to expect: do not expect quick action after the bisacodyl; do not stray far from a bathroom for several hours after taking the Pico-Salax; and, if you are taking the Pico-Salax at 22:00, do not expect to get much sleep.

Department of Medicine, University of Calgary, Calgary, Alberta

Correspondence: Dr Robert J Hilsden, Department of Medicine, University of Calgary, 3330 Hospital Drive Northwest, Calgary, Alberta T2N 4N1 .

Telelphone 403-210-9355, fax 403-283-6151, e-mail rhilsden@ucalgary.ca

Received for publication November 4, 2011. Accepted November 7, 2011 


\section{WHAT OTHER LESSONS CAN WE LEARN FROM THESE STUDIES?}

First, these studies show that split-dose preparations are the new standard of care. For an afternoon procedure, it is clearly no longer acceptable to take all of the preparation the day before. The question is, how early into the day can this be pushed? Kao et al used a split-dose regimen for patients scheduled at 12:30, while Vanner and Hookey set the cut-off at 11:00. Both groups had the patients take the second dose of preparation at 06:00. At our centre, we use a 10:00 cut-off, and ask the patients to take the second dose of preparation $5 \mathrm{~h}$ before the time of their appointment. For the 10:00 patients, this means taking the dose at 05:00. However, others have suggested that this could be pushed to even earlier in the day, with patients waking at 03:00 or 04:00 to take the preparation (4). Our experience is that it can be difficult to fill the 10:00 appointment spots. Our screening patients, who may feel less urgency to undergo a colonoscopy, are often more interested in waiting a week or two for a more appealing appointment time. Therefore, I have usually silently scoffed at the idea of asking patients to willingly get up at 04:00 to take their bowel preparation, believing it would lead to scheduling chaos and morning no shows. However, consider what Hookey and Vanner have shown us: patients are currently getting up - unwillingly - all night long when they take their prep at 22:00. So, perhaps it is all in the marketing: do you want to get $6 \mathrm{~h}$ of sleep and then take your prep, or do you want to take your prep and then get no sleep?

Second, these studies show that we still do not have the ideal solution for morning procedures. Most preparations provide a similar level of cleansing and, going forward, the road to improvement in bowel preparation quality may reside with how the preps are administered. Conceptually, one could divide colon preparation into three phases: evacuation of formed stool from the distal colon; flushing out liquid stool from the proximal colon and off of the mucosal surface; and maintaining a clean mucosal surface until the time of the colonoscopy. In regimens using bisacodyl, the first phase is the task of the bisacodyl, with the liquid preparation taking care of the rest. I believe current bowel prep regimens generally do well with the former two phases, but fail with the latter. The most common bowel prep problem, especially with morning procedures, is not residual solid stool, but that thick, tenacious, bile-stained mucous that coats the right colon. It is very difficult and time consuming to wash off and, although it would be unlikely to result in a missed cancer, it would be easy to miss a sessile serrated adenoma. Clearly, a preparation taken within a few hours of the colonoscopy is required to prevent this, but what volume is required? Is it necessary to take one-half of the preparation at 03:00 to have an excellently prepared colon at 08:00? Is it possible to take a smaller amount later in the morning? Would a smaller amount, for example, $300 \mathrm{~mL}$ to $500 \mathrm{~mL}$ of PEG taken $2 \mathrm{~h}$ to $3 \mathrm{~h}$ before the colonoscopy, be adequate to dilute intestinal secretions and prevent them from adhering to the right colon?

Are there other aspects of the bowel preparation regimen that could be 'tweaked' for better performance? A clear fluid diet the day before the colonoscopy is standard and, often if people have an inadequate bowel preparation at one colonoscopy, they are told to extend the period of clear fluids to $36 \mathrm{~h}$ to $48 \mathrm{~h}$. But is this really helpful? Several studies suggest that it is not (5-7). For example, Soweid et al (7) allowed patients to consume a fibre-free diet on the day before colonoscopy and found that it resulted in a better quality preparation. This appeared to be due to the fact that people on a low-fibre diet tolerated the bowel preparation better than those who had been consuming only liquids. Therefore, future research should address other aspects of the bowel preparation regimen, including timing and diet, to investigate further improvements in cleansing and tolerance.

Adjunctive agents may also be useful. Stengel and Jones (8) found a marked decrease in the proportion of patients with a poor bowel preparation by adding a single dose of lubiprostone (Amitiza [Takeda Pharmaceuticals America Inc, USA]) to a split-dose PEG regimen. Patients receiving lubiprostone experienced fewer symptoms with the PEG and tolerated the preparation better overall. Could the prosecretory effects of lubiprostone help prevent coating of the right colon?

\section{WHAT IF CURRENT PREPARATIONS ARE AS GOOD AS THEY ARE GOING TO GET?}

Are there ways that poor bowel preps, especially those isolated to the right colon, could be more effectively dealt with at the time of colonoscopy? Currently, my only option to address a tenacious mucous coating of the right colon is to blast it with water in the hopes that it comes off. Could a mucolytic or other agent be added to the water to help dissolve the coating?

One final area deserving more study is the risk of complications associated with the different preparations. Current studies, which usually recruit approximately 200 or fewer to each study arm, are underpowered to detect uncommon but serious adverse events. Larger studies are needed to better understand the risk of rare but serious adverse events such as ischemic colitis with bisacodyl, syncope and falls with small volume preparations, and cardiovascular complications with all preparations. Clearly, there is still much more work for the bowel prep researcher to accomplish.

\section{REFERENCES}

1. Seeff LC, Richards TB, Shapiro JA, et al. How many endoscopies are performed for colorectal cancer screening? Results from CDC's survey of endoscopic capacity. Gastroenterology 2004;127:1670-7.

2. Kao D, Lalor E, Sandha G, et al. A randomized controlled trial of four precolonoscopy bowel cleansing regimens. Can J Gastroenterol 2011;25:657-62.

3. Vanner S, Hookey LC. Timing and frequency of bowel activity in patients ingesting sodium picosulfate/magnesium citrate and adjuvant bisacodyl for colon cleansing before colonoscopy. Can J Gastroenterol 2011;25:663-6.

4. Unger RZ, Amstutz SP, Seo dH, Huffman M, Rex DK. Willingness to undergo split-dose bowel preparation for colonoscopy and compliance with split-dose instructions. Dig Dis Sci 2010;55:2030-4

5. Aoun E, Abdul-Baki H, Azar C, et al. A randomized single-blind trial of split-dose PEG-electrolyte solution without dietary restriction compared with whole dose PEG-electrolyte solution with dietary restriction for colonoscopy preparation. Gastrointest Endosc 2005;62:213-8.

6. Wu KL, Rayner CK, Chuah SK, Chiu KW, Lu CC, Chiu YC. Impact of low-residue diet on bowel preparation for colonoscopy. Dis Colon Rectum 2011;54:107-12.

7. Soweid AM, Kobeissy AA, Jamali FR, et al. A randomized singleblind trial of standard diet versus fiber-free diet with polyethylene glycol electrolyte solution for colonoscopy preparation. Endoscopy 2010;42:633-8.

8. Stengel JZ, Jones DP. Single-dose lubiprostone along with split-dose PEG solution without dietary restrictions for bowel cleansing prior to colonoscopy: A randomized, double-blind, placebo-controlled trial. Am J Gastroenterol 2008;103:2224-30. 


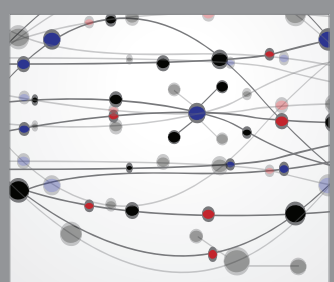

The Scientific World Journal
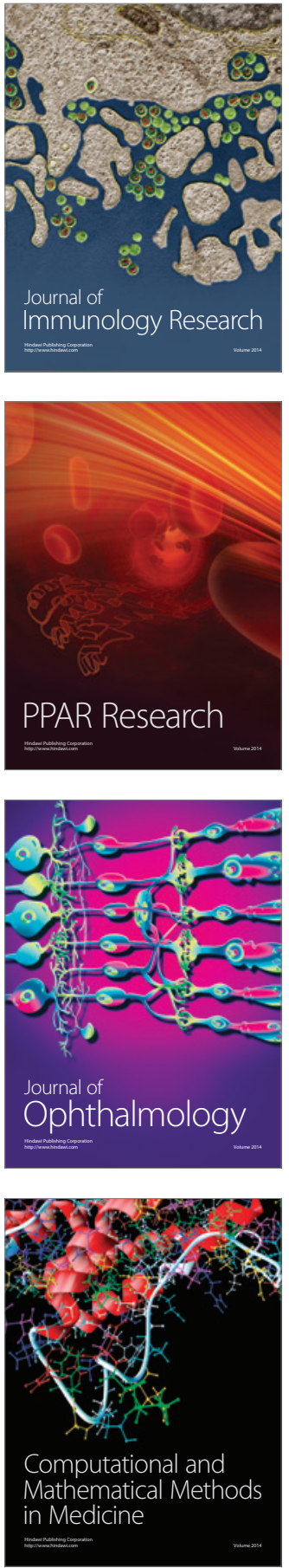

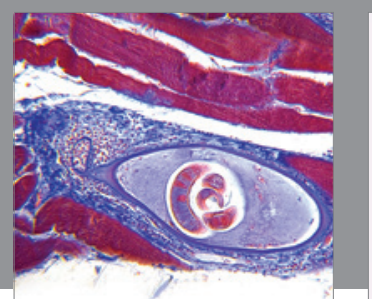

Gastroenterology Research and Practice

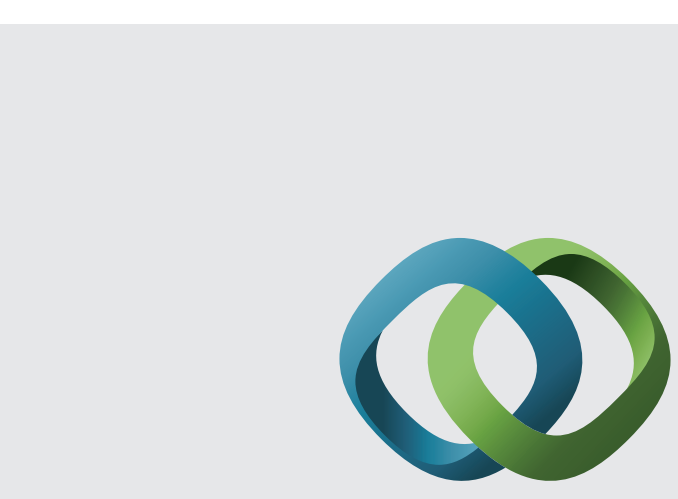

\section{Hindawi}

Submit your manuscripts at

http://www.hindawi.com
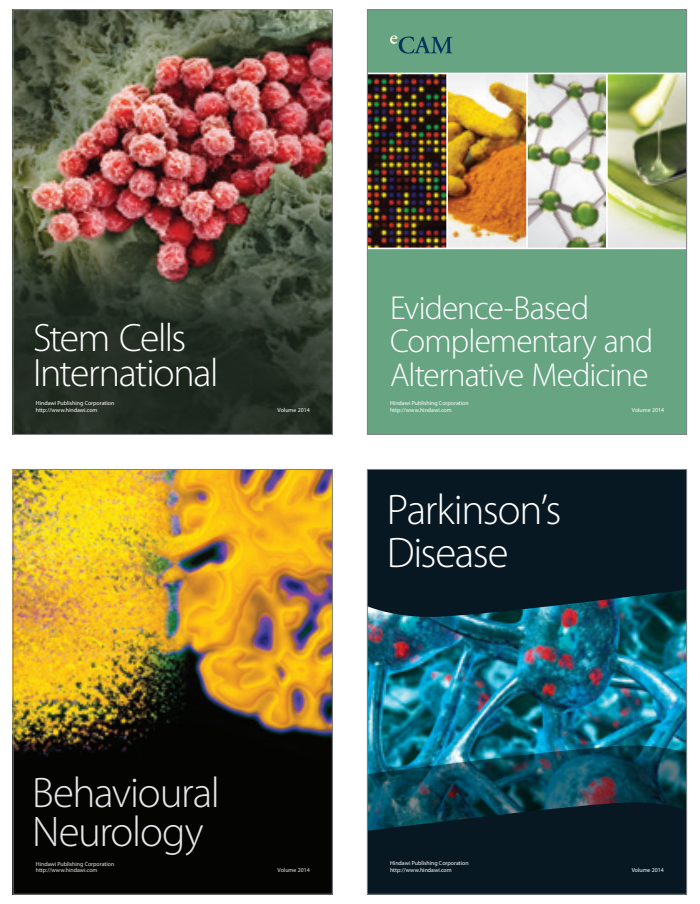
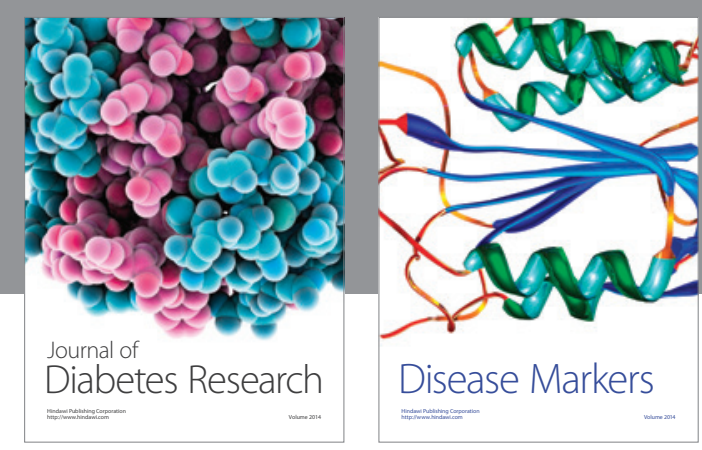

Disease Markers
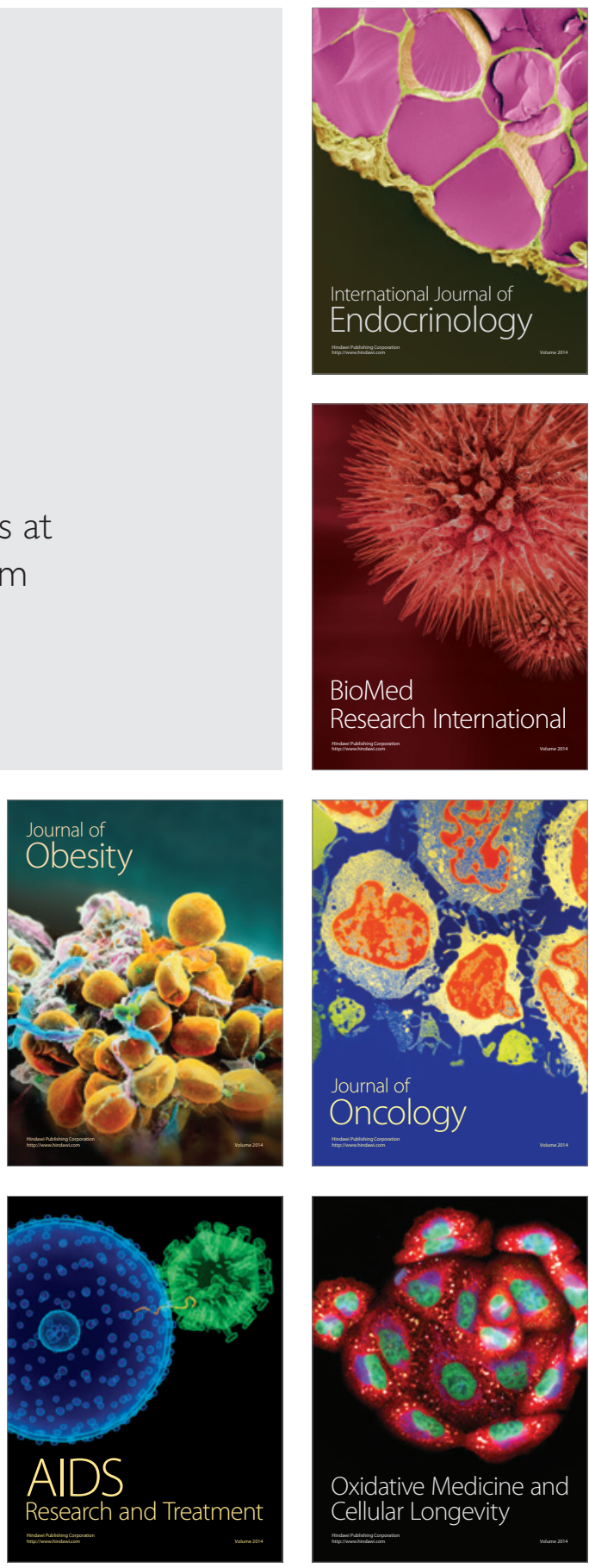